\title{
Effect of Hua Yu Xiao Zheng decoction on the expression levels of vascular endothelial growth factor and angiopoietin-2 in rats with endometriosis
}

\author{
ZHEN-ZHEN CHEN $^{1}$ and XIN GONG ${ }^{2}$ \\ ${ }^{1}$ School of Traditional Chinese Medicine, Beijing Key Lab of TCM Collateral Disease Theory Research, \\ Capital Medical University, Beijing 100069; ${ }^{2}$ Department of Gynecology, Dong Fang Hospital \\ of Beijing University of Chinese Medicine, Beijing 100078, P.R. China
}

Received August 25, 2016; Accepted May 5, 2017

DOI: $10.3892 / \mathrm{etm} .2017 .5280$

\begin{abstract}
The aims of the present study were to investigate the effects of a traditional Chinese medicine, Hua Yu Xiao Zheng (HYXZ) decoction, on surgically induced endometriosis in a rat model and to determine the possible underlying regulatory mechanisms. A total of 108 female Sprague-Dawley rats were divided into the control group $(\mathrm{n}=12)$ and endometriosis group (EM group; $n=96$ ), in which endometriosis was surgically induced in model rats by autotransplantation of endometrial tissues and 72 rats survived. After 3 weeks, the EM model rats were randomly divided into four subgroups $(\mathrm{n}=18)$, including the untreated model group, and three groups administered 7,14 or $21 \mathrm{~g} / \mathrm{kg}$ HYXZ decoction. Following 28 days of treatment, the associated proteins and genes of ectopic endometrial tissues were analyzed using immunohistochemistry, western blotting and quantitative polymerase chain reaction to investigate the underlying mechanisms. Compared with the model group, the size of the endometriotic implants decreased significantly in the HYXZ-treated groups. Furthermore, the expression levels of vascular endothelial growth factor (VEGF) and angiopoietin-2 (Ang-2) were significantly decreased in HYXZ-treated groups compared with the model group. These results indicate that HYXZ affected the inhibition of angiogenesis and decreased the endometriotic implant volumes and histopathological scores. The effectiveness of HYXZ may be partially attributed to the decrease of VEGF and Ang-2 expression levels in the ectopic endometrium.
\end{abstract}

Correspondence to: Dr Xin Gong, Department of Gynecology, Dong Fang Hospital of Beijing University of Chinese Medicine, 6 Fang Xing Yuan Community, Fang Zhuang Street, Fengtai, Beijing 100078, P.R. China

E-mail: zhongxiyigongxin@163.com

Key words: endometriosis, vascular endothelial growth factor, angiopoietin-2, Hua Yu Xiao Zheng decoction, western blotting

\section{Introduction}

Endometriosis is defined as the growth of functional endometrial tissue outside the uterine cavity, and is characterized by the presence of chronic pelvic pain and infertility in females of a reproductive age (1). This disease affects women in the 15-49 years age group and presents a serious clinical problem with reproductive consequences. Current treatments for endometriosis, including surgery and hormonal therapy, are often insufficient, and result in a high rate of relapse and various side effects, such as hepatic injury and osteoporosis (2). Furthermore, the pathogenesis of endometriosis has not yet been fully elucidated; however, the most widely-accepted hypothesis involves retrograde menstruation, which was initially proposed by Sampson in 1927 (3).

Recent studies have demonstrated the importance of neovascularization within the peritoneal cavity for endometriosis pathogenesis. Endometriotic lesions require an adequate blood supply to survive in their ectopic sites (4). Therefore, angiogenesis has become a promising target candidate for endometriosis therapy. Vascular endothelial growth factor (VEGF) is a heparin-binding glycoprotein with potent angiogenic, endothelial cell-specific mitogenic and vascular permeability activities (5). The concentration of VEGF is increased in human endometrium, and it may be important in physiological and pathological angiogenesis (6). VEGF expression varies among different types of lesions, with the early and highly vascularized red lesions exhibiting a greater VEGF expression in comparison with the later-stage and more inactive black lesions (7). In eutopic as well as ectopic endometria, no significant cyclic variations were observed throughout the cycle (7). However, VEGF content was found to be higher in the eutopic glandular epithelium of women with endometriosis during the late secretory phase, possibly suggesting a greater chance of implantation (7). Following the attachment phase, the high VEGF levels may provoke an increase in the subperitoneal vascular network, facilitating implantation and viability in the retroperitoneal space (8).

Neovascularization is considered to be a key factor in the progression of endometriosis; this pathological condition has been defined as an angiogenic disease (9). The angiopoietin 
(Ang) family of growth factors is known to mainly promote vessel maturation and remodeling (10). Ang-2 expression in the ectopic and eutopic endometria serves an important role in the pathogenesis and development of endometriosis (11). Ang-2 upregulates the production of proteases, including matrix metalloproteinases (MMPs) (12) in the presence of VEGF. Increased proteolytic activity due to MMPs may help to explain the invasive factors that result in endometriosis (13). Furthermore, Ang-2 promotes vessel sprouting in conjunction with VEGF by blocking the stabilization signal of Ang-1 at the level of Ang-1's endothelial cell-specific tyrosine kinase receptor (Tie2) (14). This suggests that there is an association between VEGF and Ang-2.

In traditional Chinese medicine (TCM), endometriosis is considered to be a syndrome caused by deficiency of the kidney with blood stasis (15). Treatment of endometriosis using TCM is common in China, and considerable research has been conducted on the role of such formulas in promoting fertility, alleviating pain and preventing relapse $(16,17)$. Hua $\mathrm{Yu}$ Xiao Zheng (HYXZ) decoction (Table I), a Chinese medicinal formula for endometriosis treatment, is prescribed based on our clinical experience. HYXZ contains Salvia miltiorrhizae radix (10.7\%), Morindae officinalis radix (7.2\%), Panax notoginseng radix (6.4\%), Semen coicis (17.9\%), Fritillariae thunberg bulbus (7.2\%), Spica prunellae (10.7\%), Polygoni aviculare herba (10.7\%), Panta rhei radix (3.6\%), Alternaria dianthi herba (10.7\%), Corydalis sp. rhizome (7.1\%), Hirudo sp. (2.1\%), Typhae sp. pollen (4.3\%) and Draconis sanguis (1.4\%). In clinical practice, it has been observed that HYXZ can alleviate endometriosis-associated symptoms, including severe dysmenorrhea, dyspareunia, menstrual irregularities and infertility. However, the mechanisms underlying the effect of HYXZ decoction on endometriosis remain unknown.

In the present study, the effects of HYXZ on the expression levels of VEGF and Ang-2 in endometriosis tissues were investigated in a rat model, in an attempt to clarify the mechanisms underlying the action of HYXZ in the treatment of endometriosis.

\section{Materials and methods}

Animals. Specific-pathogen-free grade female, nonpregnant Sprague-Dawley rats ( $\mathrm{n}=108$; body weight, $190-230 \mathrm{~g}$ ) were obtained from Vital River Laboratory Animal Technology Co., Ltd (Beijing, China; SCXK 2012-0001). Animals were raised at a constant temperature of $25^{\circ} \mathrm{C}, 50 \%$ humidity and 12 -h light/dark cycles. Standard rat feed and water were provided ad libitum. All rats were acclimatized for 7 days to ascertain health before the experiments were performed. All procedures described in the present study were reviewed and approved by the Ethical Committee of Capital Medical University (Beijing, China; approval no. AEEI-2015-100).

Preparation of formula. All the medicinal plants used to prepare the HYXZ decoction were obtained from the Pharmacy Department of Dong Fang Hospital of Beijing University of Chinese Medicine (Beijing, China). The quality of the raw herbs was controlled according to the Pharmacopoeia of the People's Republic of China (18). The aqueous extract of HYXZ was prepared according to a previously described procedure (19). Briefly, the mixture of herbs listed in Table I was macerated in distilled water for $0.5 \mathrm{~h}$. Following this, the herbs were decocted for $0.5 \mathrm{~h}$, and the residue was then decocted again for $0.5 \mathrm{~h}$. The final extract was subsequently filtered, combined and concentrated to $2.1 \mathrm{~g} / \mathrm{ml}$ (crude dosage) by heating.

Rat model of endometriosis. The animal model was established by autotransplantation of endometrial tissues, as previously reported by Zhang et al (20). Briefly, 96 rats were anesthetized with intraperitoneal administration of $10 \%$ chloral hydrate $(\mathrm{m} / \mathrm{v})$ at a dose of $0.35 \mathrm{ml} / 100 \mathrm{~g}$ body weight. Prior to surgery, the abdominal skin was disinfected with $75 \%$ ethanol and the abdominal cavity was opened. The uterine vessel was ligated with 5-0 polypropylene sutures. The right uterine horn was removed and placed in a Petri dish containing phosphate-buffered saline (PBS) at $37^{\circ} \mathrm{C}$. Approximately $1 \mathrm{~cm}$ of the uterine horn was cut along the longitudinal axis, and then divided in half. Two squares of $5 \times 5 \mathrm{~mm}$ open uterus were prepared. Each endometrium segment was fixed with 6-0 sutures to the peritoneal side of the bilateral abdominal wall with the endometrium facing the abdominal wall. Subsequently, the abdominal muscle and skin was closed using 4-0 polypropylene sutures. The control group underwent sham surgery, consisting solely of the unilateral hemihysterectomy without autotransplantation of endometrial tissues. Following surgery, all rats were treated with cephalosporin ( $0.1 \mathrm{~g}$; Tianjin Pharmaceutical Holdings Gencom Pharmacy Co., Ltd., Tianjin, China) by intraperitoneal injection for 3 days. After a recovery period of 21 days, the endometriosis model rats underwent a second exploratory laparotomy to examine whether the models of experimental endometriosis had been successfully established, which was determined by observation of red/brown color and cystic formation on the implant surfaces (21). In the present experiment, 72 out of 96 model rats survived, with a success percentage of $75 \%$, and these rats were used in subsequent experiments.

Treatment. The 72 rats with successful endometriosis model were randomly divided into four groups as follows ( $\mathrm{n}=18$ in each group): Endometriosis (EM group), endometriosis + low-dose HYXZ (EM + L group), endometriosis + mid-dose HYXZ (EM + M group), endometriosis + high-dose HYXZ (EM + H group). Rats in the $\mathrm{EM}+\mathrm{L}, \mathrm{EM}+\mathrm{M}$ and $\mathrm{EM}+\mathrm{H}$ groups were administered with HYXZ at a dose of 7, 14 and $21 \mathrm{~g} / \mathrm{kg} / \mathrm{day}$, respectively. The mid-dose group, which was similar to the dose administered to female patients, was calculated using the formula of dose translation among different species based on body surface area (22). Rats in the control and EM groups were administered with double-distilled water $(10 \mathrm{ml} / \mathrm{kg})$. The drugs and double-distilled water were administered daily by oral gavage for 28 days.

Specimen collection. After treatment for 28 days, the rats were sacrificed. Ectopic endometrial tissues were measured and dissected from the implant sites. Half of the lesion was immediately fixed in $4 \%$ paraformaldehyde and then embedded in paraffin for hematoxylin-eosin (HE) staining and immunohistochemical assays. The remaining half of the lesion was stored at $-80^{\circ} \mathrm{C}$ for analysis of the protein and mRNA levels of VEGF and Ang-2. 
Table I. Composition of Hua Yu Xiao Zheng decoction.

\begin{tabular}{llr}
\hline Herb & \multicolumn{1}{c}{ Component } \\
\hline Danshen & Salviae miltiorrhizae Bunge, root & 15 \\
Bajitian & Morinda officinalis How, root & 10 \\
Sanqi & Panax notoginseng (Burk.) F.H. Chen, root & 9 \\
Yiyiren & Coix lacryma-jobi L. var. ma-yuen (Roman.) Stapf, seed \\
Zhebeimu & Fritillaria thunbergii Miq., tuber \\
Xiakucao & Prunella vulgaris L., cluster \\
Bianxu & Polygonum aviculare L., overground part \\
Dahuang & Rheum palmatum L., root \\
Qumai & Dianthus superbus L., overground part \\
Yanhusuo & Corydalis yanhusuo W.T. Wang, tuber \\
Shuizhi & Whitmania pigra Whitman, whole body \\
Puhuang & Typha angustifolia L., pollen \\
Xuejie & Daemonorops draco Bl., resin \\
Total amount & & 10 \\
\hline
\end{tabular}

$H \& E$ staining. Both eutopic endometrium and ectopic endometria of rats were surgically detached. The endometriotic implants were first fixed in $10 \%$ formaldehyde solution, embedded in paraffin and cut into $4-\mu \mathrm{m}$ sections. These sections were stained withH\&Eand examined under a light microscope. The morphology was captured with a digital camera (Nikon 4500; Nikon Corp., Tokyo, Japan).

Immunohistochemical analysis. The paraffin-embedded sections of ectopic endometrial tissue were subjected to antigen retrieval with citric acid buffer, and were then incubated overnight at $4^{\circ} \mathrm{C}$ with the following primary antibodies: VEGF antibody (Ab46154; dilution, 1:200; Abcam, Cambridge, UK); and Ang-2 antibody (Ab180820; dilution, 1:200; Abcam). Subsequent to washing in PBS (pH 7.4) three times, the sections were incubated with secondary antibody (Ab205718; dilution, 1:50; Abcam) for $60 \mathrm{~min}$ at $37^{\circ} \mathrm{C}$. Following incubation, the slides were rewashed with PBS and incubated with $0.01 \%$ 3,3-diaminobenzidine tetrahydrochloride hydrate for $\sim 1 \mathrm{~min}$. Sections were then washed thoroughly in PBS three times for $5 \mathrm{~min}$ each, and counterstained in hematoxylin for $20 \mathrm{sec}$. Finally, the tissues were dehydrated, rendered transparent and mounted prior to being analyzed under a microscope. Negative control tissues were treated with the same procedure, using PBS instead of primary antibody. Images of the tissues were captured by a digital camera (Nikon 4500). The positive area and optical density (OD) of positive cells were determined by measuring three randomly selected microscopic fields for each slide. The immunohistochemical index was defined as the average integral OD (AIOD), as follows: $\mathrm{AIOD}=$ positive area $\mathrm{x}$ OD/total area (23).

Western blot analysis. The tissues of the ectopic endometria were sectioned and lysed in radioimmunoprecipitation assay lysis buffer (C1053; Applygen Technologies, Inc., Beijing, China). The concentration of protein used for western blot analysis was first quantified by a bicinchoninic acid assay (P1511; Applygen Technologies, Inc.). Next, protein samples
$(20 \mu \mathrm{g})$ were subjected to $10 \%$ sodium dodecyl sulfate-polyacrylamide gel electrophoresis and then transferred to a polyvinylidene difluoride (PVDF) membrane. The membrane was blocked for $1 \mathrm{~h}$ at room temperature in $5 \%$ skimmed milk. Subsequent to blocking, the membranes were incubated at $4^{\circ} \mathrm{C}$ overnight with the following primary antibodies: VEGF (Ab46154; dilution, 1:2,000), Ang-2 (Ab180820; dilution, 1:2,000) and GAPDH (Ab181602; dilution, 1:20,000). All the primary antibodies were obtained from Abcam. Following rinsing in Tris-Buffered Saline and Tween-20 three times, the PVDF membranes were incubated with the secondary antibodies (C1633; dilution, 1:2,000; Applygen Technologies, Inc.) at room temperature for $1 \mathrm{~h}$. Images were obtained using electrochemiluminescence (NCI4106; Pierce; Thermo Fisher Scientific, Inc., Waltham, MA, USA), prior to being analyzed using Image J software v1.46r (National Institutes of Health, Bethesda, MD, USA).

Reverse transcription-quantitative polymerase chain reaction (RT-qPCR) analysis. Total RNA was isolated from ectopic endometrial tissues using TRIzol reagent (15596-026; Thermo Fisher Scientific, Inc.), and then reverse transcribed into cDNA with a Reverse Transcription kit (D6130; Takara Bio, Inc., Otsu, Japan). The resulting cDNA was diluted 10-fold in sterile water, and aliquots were subjected to qPCR. The PCR primer pairs used in the analysis were designed and synthetized by Taihe Biotechnology Co., Ltd. (Beijing, China; Table II). PCR cycles were composed of one cycle of $95^{\circ} \mathrm{C}$ for $5 \mathrm{~min}$ to denature all proteins, 40 cycles of $95^{\circ} \mathrm{C}$ for $20 \mathrm{sec}$, and then $65^{\circ} \mathrm{C}$ for $30 \mathrm{sec}$. The reaction was terminated at $72^{\circ} \mathrm{C}$ for $5 \mathrm{~min}$ and quenched at $4^{\circ} \mathrm{C}$. qPCR reactions were performed with an AMI Prism 7700 sequence detection system (Applied Biosystems; Thermo Fisher Scientific, Inc.). The relative expression of each target gene compared with GAPDH was analyzed using the $2^{-\Delta \Delta \mathrm{Cq}}$ method (24).

Statistical analysis. All statistical analyses were performed using the SPSS software, version 17.0 (SPSS, Inc., Chicago, IL, 
Table II. Quantitative polymerase chain reaction primer sequences.

\begin{tabular}{llc}
\hline Gene & \multicolumn{1}{c}{ Primer sequences $\left(5^{\prime} \rightarrow 3^{\prime}\right)$} & $\begin{array}{c}\text { Product } \\
\text { size }(\mathrm{bp})\end{array}$ \\
\hline VEGF & $\begin{array}{l}\text { F: GGCTCACTTCCAGAAACACG } \\
\text { R: GTGCTCTTGCAGAATCTAGTGG }\end{array}$ & 165 \\
Ang-2 & $\begin{array}{l}\text { F: CGGACTCTGTCACAAGCAAGAA } \\
\text { R: AGCACAAGACGGAACAACGAA }\end{array}$ & 237 \\
GAPDH & $\begin{array}{l}\text { F: TGCTGAGTATGTCGTGGAG } \\
\text { R: GTCTTCTGAGTGGCAGTGAT }\end{array}$ & 288 \\
\hline
\end{tabular}

VEGF, vascular endothelial growth factor; Ang-2, angiopoietin-2; F, forward; R, reverse.

USA). Values are expressed as the mean \pm standard deviation. The analysis of variance test was used to compare the differences among the animal groups. Differences with a $\mathrm{P}<0.05$ were considered to be statistically significant.

\section{Results}

Treatment with HYXZ reduces the volume of the lesion. After the 28-day treatment with HYXZ, the longest lengths and perpendicular widths of the lesions were measured by a vernier calliper. The volume of the endometriotic lesion was calculated according to the following formula: $0.52 \mathrm{x}$ width $^{2} \mathrm{x}$ length (25). A comparison of the HYXZ-treated groups and the EM model group demonstrated a statistically significant reduction in the lesion size following the treatment $(\mathrm{P}<0.05)$. As the dose increased, the volume of the lesion size was further reduced (Fig. 1; Table III).

Light microscopy analysis. The H\&Estaining results demonstrated that the shape of the eutopic endometrium epithelial cells was columnar, and the glandular epithelial and stromal cells were normal in the control group. In the EM model group, H\&E staining revealed that the ectopic endometrium was covered with connective tissues and that the glands and intercellular substances grew well and intensively. The intima was thick and the glandular and superficial epithelia formed a high column. Evident hyperplasia and angiopoiesis were also observed. In the $\mathrm{EM}+\mathrm{L}, \mathrm{EM}+\mathrm{M}$ and $\mathrm{EM}+\mathrm{H}$ treatment groups, the columnar shape was nearly natural, while the number of glands and glandular epithelial cells was almost normal (Fig. 2).

Expression levels of VEGF and Ang-2 as evaluated by immunohistochemistry. As illustrated in Fig. 3 and Table IV, the immunohistochemical scores of VEGF and Ang-2 were higher in the EM model group compared with the control sham-operated group $(\mathrm{P}<0.05)$. Following HYXZ administration (EM $+\mathrm{L}, \mathrm{EM}+\mathrm{M}$ and $\mathrm{EM}+\mathrm{H}$ groups), the Ang-2 immunohistochemical score was significantly decreased compared with that of the EM group $(\mathrm{P}<0.05)$. Similarly, the VEGF immunohistochemical score was significantly decreased in the $\mathrm{EM}+\mathrm{H}$ group compared with the $\mathrm{EM}$ group $(\mathrm{P}<0.05)$, but not markedly altered in the $\mathrm{EM}+\mathrm{L}$ and $\mathrm{EM}+\mathrm{H}$ groups.
Table III. Volume of lesions following HYXZ decoction treatment.

\begin{tabular}{lcc}
\hline Group & Median $\left(\mathrm{mm}^{3}\right)$ & $\begin{array}{c}\text { Percentiles } \\
(\mathrm{P} 25-\mathrm{P} 75)\end{array}$ \\
\hline $\mathrm{EM}$ & 133.48 & $111.35-153.11$ \\
$\mathrm{EM}+\mathrm{L}$ & $74.75^{\mathrm{a}}$ & $63.40-92.68$ \\
$\mathrm{EM}+\mathrm{M}$ & $70.20^{\mathrm{a}}$ & $65.58-78.38$ \\
$\mathrm{EM}+\mathrm{H}$ & $26.03^{\mathrm{a}}$ & $21.79-52.88$ \\
\hline
\end{tabular}

${ }^{\mathrm{a}} \mathrm{P}<0.05$ vs. $\mathrm{EM}$ group. The $\mathrm{EM}+\mathrm{L}, \mathrm{EM}+\mathrm{M}$ and $\mathrm{EM}+\mathrm{H}$ groups were treated with 7, 14 and $21 \mathrm{~g} / \mathrm{kg} /$ day HYXZ, respectively. HYXZ, Hua Yu Xiao Zheng; EM, endometriosis.

A
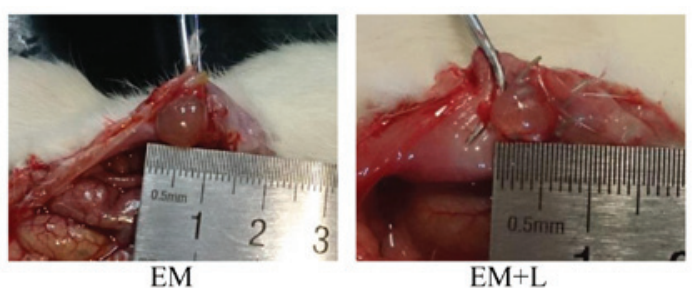

$\mathrm{EM}+\mathrm{L}$
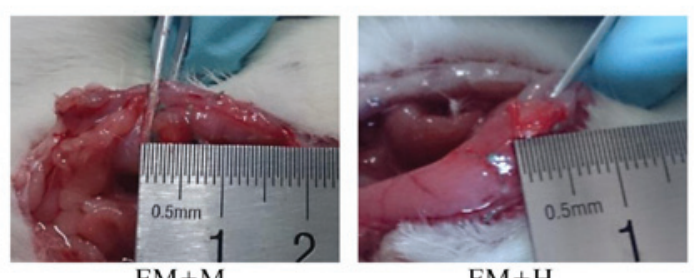

$\mathrm{EM}+\mathrm{H}$

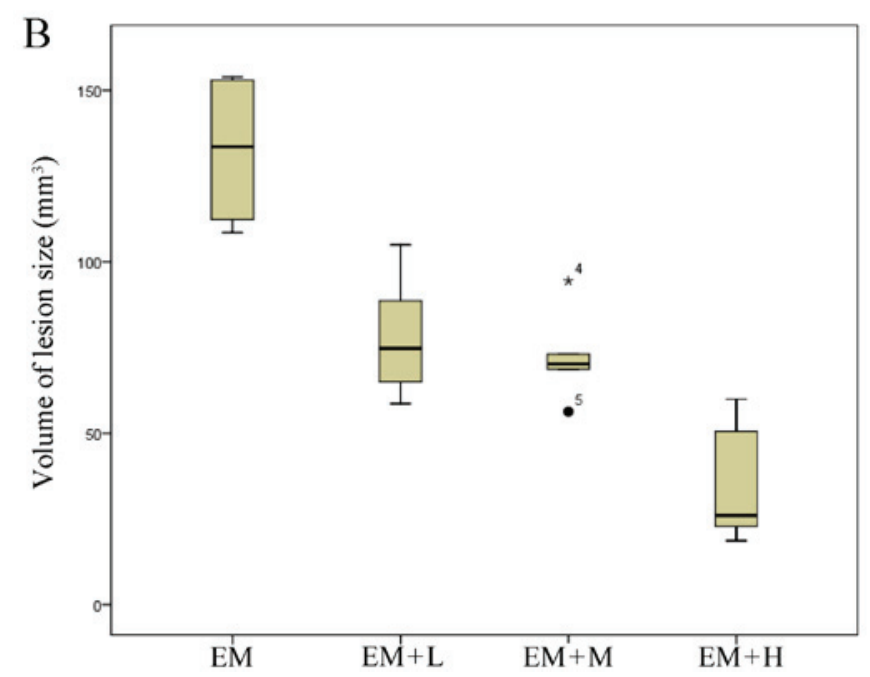

Figure 1. Volume of ectopic endometrial tissue following the treatment with different doses of the HYXZ decoction via oral gavage. (A) Comparison of the volume of the lesion in each group after 28 days of treatment. (B) Box plot demonstrating the endometrial volumes of different groups. ${ }^{*} \mathrm{P}<0.05$ The $\mathrm{EM}+\mathrm{L}, \mathrm{EM}+\mathrm{M}$ and $\mathrm{EM}+\mathrm{H}$ groups were treated with 7,14 and $21 \mathrm{~g} / \mathrm{kg} / \mathrm{day}$ HYXZ, respectively. HYXZ, Hua Yu Xiao Zheng; EM, endometriosis.

Protein levels of VEGF and Ang-2. As shown in Fig. 4 and Table V, the protein levels of VEGF and Ang- 2 in the EM group manifested a statistically significant increase in comparison with those in the control group $(\mathrm{P}<0.05)$. Compared with the 


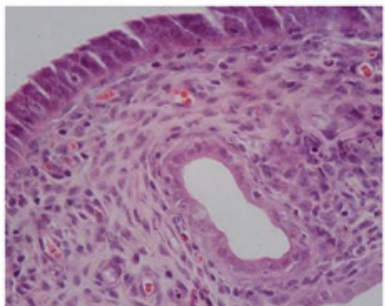

Control

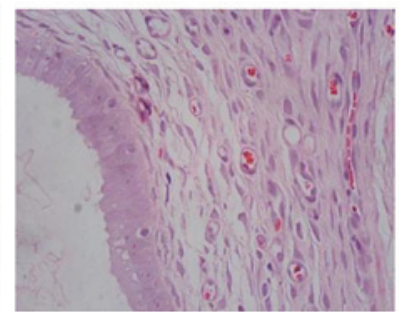

EM

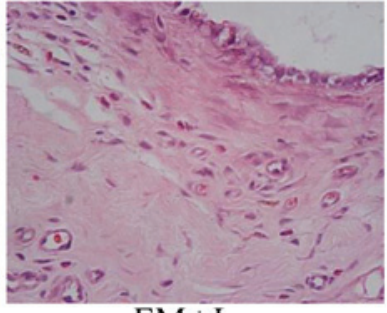

$\mathrm{EM}+\mathrm{L}$

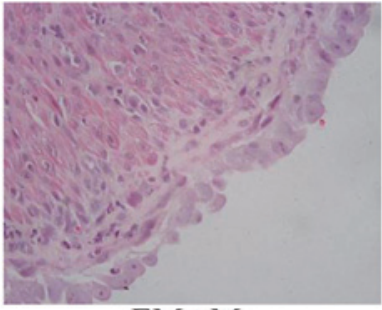

$\mathrm{EM}+\mathrm{M}$

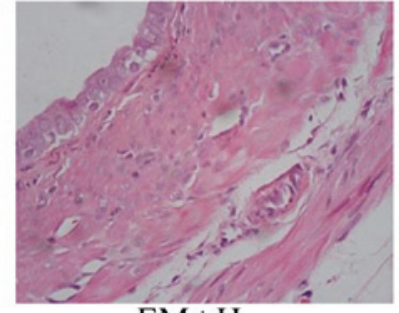

$\mathrm{EM}+\mathrm{H}$

Figure 2. Pathological morphology of the eutopic (control) and ectopic (EM groups) endometrial tissues in each group after 28 days of treatment analyzed by hematoxylin-eosin staining and light microscopy (magnification, $\mathrm{x} 40$ ). The EM + L, EM + M and EM + H groups were treated with 7, 14 and $21 \mathrm{~g} / \mathrm{kg} / \mathrm{day}$ HYXZ, respectively. HYXZ, Hua Yu Xiao Zheng; EM, endometriosis.

A

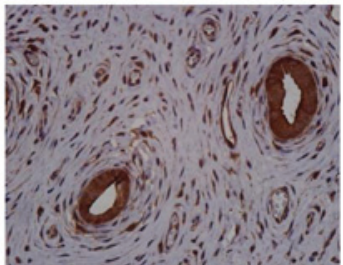

Control

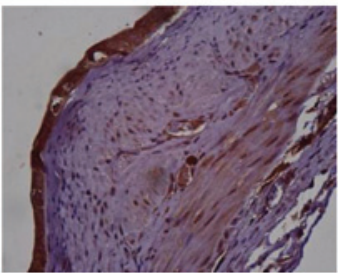

EM

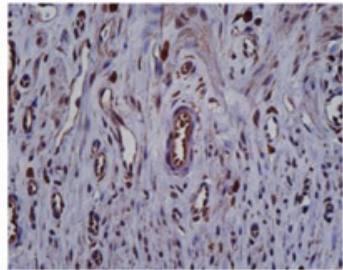

$\mathrm{EM}+\mathrm{L}$

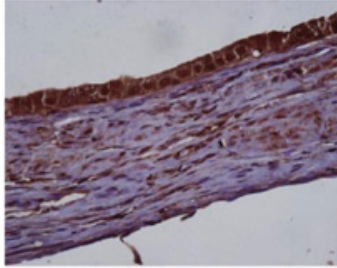

$\mathrm{EM}+\mathrm{M}$

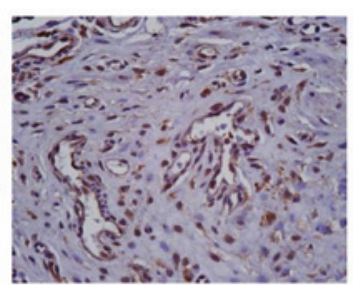

$\mathrm{EM}+\mathrm{H}$

$\mathrm{B}$

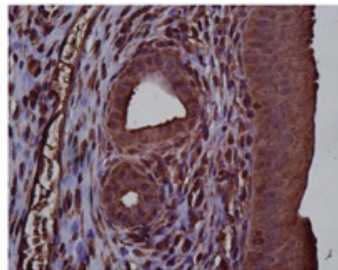

Control

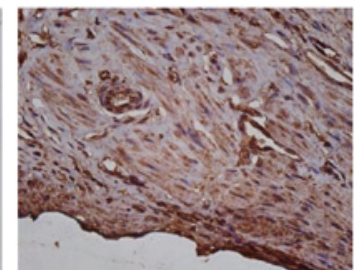

EM

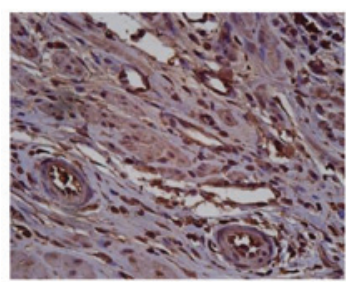

$\mathrm{EM}+\mathrm{L}$

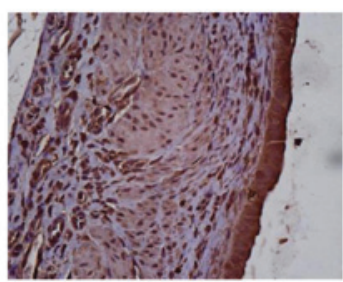

$\mathrm{EM}+\mathrm{M}$

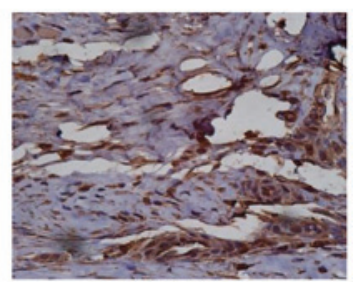

$\mathrm{EM}+\mathrm{H}$

Figure 3. Immunohistochemical analysis for the expression of (A) VEGF and (B) Ang-2 in the endometriotic lesions at the end of the treatment (magnification, $x$ 40). The EM + L, EM + M and EM + H groups were treated with 7, 14 and $21 \mathrm{~g} / \mathrm{kg} / \mathrm{day}$ HYXZ, respectively. HYXZ, Hua Yu Xiao Zheng; EM, endometriosis; VEGF, vascular endothelial growth factor; Ang-2, angiopoietin-2. 
Table IV. Scores of immunohistochemical staining for VEGF and Ang-2 in the endometriotic lesions.

\begin{tabular}{|c|c|c|}
\hline \multirow[b]{2}{*}{ Group } & \multicolumn{2}{|c|}{ AIOD } \\
\hline & VEGF & Ang-2 \\
\hline Control & $4.30 \pm 1.51$ & $2.37 \pm 0.29$ \\
\hline EM & $12.33 \pm 7.82^{\mathrm{a}}$ & $8.86 \pm 1.94^{\mathrm{a}}$ \\
\hline $\mathrm{EM}+\mathrm{L}$ & $9.05 \pm 3.22$ & $6.46 \pm 1.90^{\mathrm{b}}$ \\
\hline $\mathrm{EM}+\mathrm{M}$ & $10.66 \pm 3.65$ & $5.55 \pm 2.23^{b}$ \\
\hline $\mathrm{EM}+\mathrm{H}$ & $6.44 \pm 1.33^{\mathrm{b}}$ & $3.44 \pm 1.10^{\mathrm{b}}$ \\
\hline \multicolumn{3}{|c|}{$\begin{array}{l}\text { a }<0.05 \text { vs. control group; }{ }^{\text {b }}<0.05 \text { vs. EM group. The } \mathrm{EM}+\mathrm{L} \text {, } \\
\mathrm{EM}+\mathrm{M} \text { and } \mathrm{EM}+\mathrm{H} \text { groups were treated with } 7,14 \text { and } 21 \\
\mathrm{~g} / \mathrm{kg} / \text { day Hua } \mathrm{Yu} \text { Xiao Zheng, respectively. AIOD, average integral } \\
\text { optical density; VEGF, vascular endothelial growth factor; Ang- }, \\
\text { angiopoietin-2. }\end{array}$} \\
\hline
\end{tabular}

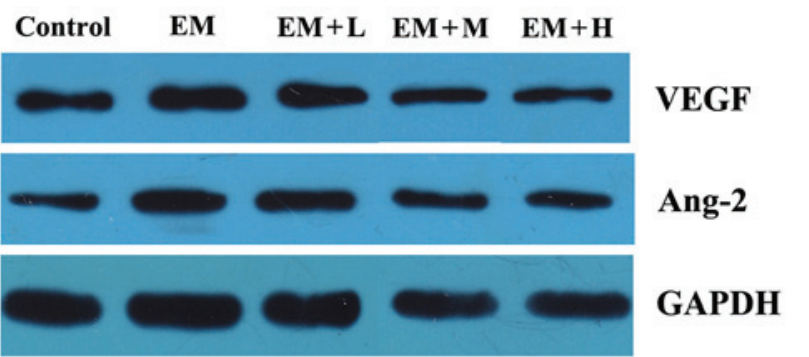

Figure 4. Effect of HYXZ decoction on the protein levels of VEGF and Ang-2, as determined by western blot analysis. $\mathrm{EM}+\mathrm{L}, \mathrm{EM}+\mathrm{M}$ and $\mathrm{EM}+\mathrm{H}$ were treated with 7, 14 and $21 \mathrm{~g} / \mathrm{kg}$ /day HYXZ, respectively. HYXZ, Hua Yu Xiao Zheng; EM, endometriosis; VEGF, vascular endothelial growth factor; Ang-2, angiopoietin-2.

EM group, the protein levels of VEGF markedly decreased in all the HYXZ-treated groups $(\mathrm{EM}+\mathrm{L}, \mathrm{EM}+\mathrm{M}$ and $\mathrm{EM}+\mathrm{H})$ in a dose-dependent manner $(\mathrm{P}<0.05)$. Furthermore, it was also observed that the protein levels of Ang-2 in the EM $+\mathrm{M}$ and $\mathrm{EM}+\mathrm{H}$ groups were significantly decreased compared with those in the EM group $(\mathrm{P}<0.05)$.

Expression of VEGF and Ang-2 $m R N A$. Results of RT-qPCR demonstrated a pronounced difference in the expression levels of VEGF and Ang-2 mRNA in the EM model and HYXZ-treated groups when compared with the control group. As shown in Table VI, the expression levels of VEGF and Ang-2 mRNA in the EM group were significantly upregulated in comparison with the control group $(\mathrm{P}<0.05)$. Furthermore, the expression of VEGF and Ang- 2 mRNA in the EM $+\mathrm{M}$ and $\mathrm{EM}+\mathrm{H}$ treatment groups was markedly downregulated in comparison with the EM model group $(\mathrm{P}<0.05)$.

\section{Discussion}

Endometriosis is a common gynecological condition with diverse clinical manifestations, as well as highly variable and unpredictable clinical course with decreased quality of life. Although endometriosis is a benign disorder, the process
Table V. Effect of Hua Yu Xiao Zheng decoction on protein levels of VEGF and Ang-2.

\begin{tabular}{lccc}
\hline Group & $\begin{array}{c}\text { Dose } \\
(\mathrm{g} / \mathrm{kg} / \text { day })\end{array}$ & $\begin{array}{c}\text { VEGF/ } \\
\text { GAPDH }\end{array}$ & $\begin{array}{c}\text { Ang-2/ } \\
\text { GAPDH }\end{array}$ \\
\hline Control & - & $0.30 \pm 0.03$ & $0.73 \pm 0.09$ \\
EM & - & $0.49 \pm 0.06^{\mathrm{a}}$ & $0.92 \pm 0.05^{\mathrm{a}}$ \\
$\mathrm{EM}+\mathrm{L}$ & 7 & $0.41 \pm 0.04^{\mathrm{b}}$ & $0.86 \pm 0.07$ \\
$\mathrm{EM}+\mathrm{M}$ & 14 & $0.38 \pm 0.03^{\mathrm{b}}$ & $0.74 \pm 0.07^{\mathrm{b}}$ \\
$\mathrm{EM}+\mathrm{H}$ & 21 & $0.36 \pm 0.02^{\mathrm{b}}$ & $0.70 \pm 0.06^{\mathrm{b}}$ \\
\hline
\end{tabular}

${ }^{\mathrm{a}} \mathrm{P}<0.05$ vs. control group; ${ }^{\mathrm{b}} \mathrm{P}<0.05$ vs. EM group. EM, endometriosis; VEGF, vascular endothelial growth factor; Ang-2, angiopoietin-2.

Table VI. Effect of Hua Yu Xiao Zheng decoction on mRNA levels of VEGF and Ang-2.

\begin{tabular}{lcll}
\hline Group & $\begin{array}{c}\text { Dose } \\
(\mathrm{g} / \mathrm{kg} / \text { day })\end{array}$ & $\begin{array}{c}\text { VEGF/ } \\
\text { GAPDH }\end{array}$ & $\begin{array}{c}\text { Ang-2/ } \\
\text { GAPDH }\end{array}$ \\
\hline Control & - & $0.76 \pm 0.24$ & $0.78 \pm 0.35$ \\
EM & - & $8.92 \pm 3.73^{\mathrm{a}}$ & $7.01 \pm 2.29^{\mathrm{a}}$ \\
$\mathrm{EM}+\mathrm{L}$ & 7 & $7.02 \pm 2.66$ & $6.59 \pm 2.47$ \\
$\mathrm{EM}+\mathrm{M}$ & 14 & $4.47 \pm 1.51^{\mathrm{b}}$ & $3.89 \pm 1.35^{\mathrm{b}}$ \\
EM + H & 21 & $3.14 \pm 0.98^{\mathrm{b}}$ & $3.32 \pm 1.19^{\mathrm{b}}$ \\
\hline
\end{tabular}

${ }^{\mathrm{a}} \mathrm{P}<0.05$ vs. control group; ${ }^{\mathrm{b}} \mathrm{P}<0.05$ vs. EM group. EM, endometriosis; VEGF, vascular endothelial growth factor; Ang-2, angiopoietin-2.

through which endometrial cells attach to and invade surface shares features of malignancy (26). Current treatments, including surgery and hormonal therapy, are often insufficient, as well as present a high rate of relapse and various side effects, such as weight gain, abnormal lipid metabolism, hepatic injury and osteoporosis. Therefore, it is necessary to explore novel medical strategies and treatment approaches to improve the treatment of endometriosis lesions.

TCM herbs have become popular for relieving endometriosis-associated symptoms, including dysmenorrhea, dyspareunia, menorrhagia and chronic pain, which typically require surgical or medical intervention $(27,28)$. Chinese herbal formulas for endometriosis therapy are designed to alleviate blood stasis and nourish the kidney (29). The present study aimed to examine the therapeutic potential of HYXZ, determine its ability to reduce endometrial explants growth and determine the possible mechanism of action in EM model rats, in order to provide a theoretical foundation for endometriosis treatment. To the best of our knowledge, the current study demonstrated for the first time that a physiological dose of HYXZ (which is six times greater than the amount administered to humans) administered for 28 days inhibited the growth of endometriotic tissues in a dose-dependent manner. Furthermore, the results demonstrated that HYXZ did not suppress the growth of the eutopic endometrium inside the uterine cavity of the rat model. These findings indicate 
that HYXZ specifically targets the endometriotic tissues with minimal side effects on normal endometrium.

The development of new blood vessels is a complex dynamic process, which is characterized by a coordinated sequence of humoral and cellular interactions. It is widely accepted that endometriotic lesions are typically characterized by a dense vascularization that occurs through the angiogenesis process $(30,31)$. The establishment and development of an effective blood supply and angiogenesis are essential for the growth of endometriotic implants (32). Endometrial expression of VEGF, which is a potent vascular permeability-inducing, mitogen and morphogen agent, is thus an important mediator of angiogenesis (33). Furthermore, Ang proteins are potent angiogenic factors that are expressed in the normal human endometrium. Ang-2 activates in response to hypoxia and induces vessel destabilization upon binding the Tie2 receptor (34). VEGF and Ang-2 have been reported to independently induce angiogenesis (35). Furthermore, they have been demonstrated to act synergistically to induce endothelial destabilization, increase vascular branching, and increase angiogenesis (36). In addition, the two growth factors independently induce formation of endothelial branches in ex vivo aortic ring assays, suggesting an angiogenic effect on perivascular cells as well (37).

Endometriotic lesions are highly vascularized, and angiogenesis is considered as a critical step for the establishment of endometriosis $(38,39)$. VEGF stimulates endothelial cell proliferation and migration, while elevated levels of VEGF have been reported in the peritoneal fluid of women with endometriosis (7), with the same increase observed for Ang-2. In the present study, the protein and mRNA expression levels of VEGF and Ang-2 were significantly higher in the ectopic endometrium as compared with those in the normal endometrium. Thus, these results suggest that VEGF and Ang-2 serve an important role in the pathogenesis of endometriosis. The findings of the present study offer a novel perspective on the underlying mechanisms of endometriosis and indicate that anti-angiogenic therapy may be a potentially promising treatment method for patients with endometriosis. Furthermore, the current study observed that HYXZ was able to significantly reduce the protein and mRNA expression of VEGF and Ang-2 in the ectopic endometrium. The results also indicated that inhibition of angiogenesis may lead to regression of the lesion size following HYXZ treatment. Compared with other TCM formulas previously reported, HYXZ reduced the volume of endometriosis lesions to a similar extent as Guizhi Fuling capsules (40), and also demonstrated a significant inhibition of the angiogenesis process.

Although the present study offered a unique evaluation of the effects of HYXZ decoction on the endometriosis model, the small number of animals in the groups is a limitation of the study. Larger prospective controlled clinical trials are required in order to further elucidate the findings.

In conclusion, the current study revealed that $\mathrm{HYXZ}$ decoction significantly reduced the size of the endometrial explants in endometriosis model rats by decreasing the protein and mRNA levels of VEGF and Ang-2 in the ectopic endometrium. HYXZ may be a promising TCM formula to treat endometriosis, however, further clinical trials are required to confirm its efficacy.

\section{References}

1. Burney RO and Giudice LC: Pathogenesis and pathophysiology of endometriosis. Fertil Steril 98: 511-519, 2012.

2. Jacobson TZ, Duffy JM, Barlow DH, Koninckx PR and Garry R: WITHDRAWN: Laparoscopic surgery for pelvic pain associated with endometriosis. Cochrane Database Syst Rev: CD001300, 2014.

3. Sampson JA: Metastatic or embolic endometriosis, due to the menstrual dissemination of endometrial tissue into the venous circulation. Am J Pathol 3: 93-110.43, 1927.

4. Groothuis PG, Nap AW, Winterhager E and Grümmer R: Vascular development in endometriosis. Angiogenesis 8: 147-156, 2005.

5. Mueller MD, Vigne JL, Minchenko A, Lebovic DI, Leitman DC and Taylor RN: Regulation of vascular endothelial growth factor (VEGF) gene transcription by estrogen receptors alpha and beta. Proc Natl Acad Sci USA 97: 10972-10977, 2000.

6. Rocha AL, Reis FM and Taylor RN: Angiogenesis and endometriosis. Obstet Gynecol Int 2013: 859619, 2013.

7. Donnez J, Smoes P, Gillerot S, Casanas-Roux F and Nisolle M: Vascular endothelial growth factor (VEGF) in endometriosis. Hum Reprod 13: 1686-1690, 1998.

8. Charnock-Jones DS, Sharkey AM, Rajput-Williams J, Burch D, Schofield JP, Fountain SA, Boocock CA and Smith SK: Identification and localization of alternately spliced mRNAs for vascular endothelial growth factor in human uterus and estrogen regulation in endometrial carcinoma cell lines. Biol Reprod 48: 1120-1128, 1993.

9. Healy DL, Rogers PA, Hii L and Wingfield M: Angiongenesis: A new theory for endometriosis. Hum Reprod Update 4: 736-740, 1998.

10. Yancopoulos GD, Davis S, Gale NW, Rudge JS, Wiegand SJ and Holash J: Vascular-specific growth factors and blood vessel formation. Nature 407: 242-248, 2000.

11. Jingting C, Yangde Z, Yi Z, Mengxiong L, Rong Y, Yu Z, Guoqing $P$ and Lixiu P: Expression of heparanase and angiopoietin-2 in patients with endometriosis. Eur J Obstet Gynecol Reprod Biol 136: 199-209, 2008.

12. Loukovaara S, Robciuc A, Holopainen JM, Lehti K, Pessi T, Liinamaa J, Kukkonen KT, Jauhiainen M, Koli K, Keski-Oja J and Immonen I: Ang-2 upregulation correlates with increased levels of MMP-9, VEGF, EPO and TGF $\beta 1$ in diabetic eyes undergoing vitrectomy. Acta Ophthalmol 91: 531-539, 2013.

13. Weigel MT, Krämer J, Schem C, Wenners A, Alkatout I, Jonat W, Maass N and Mundhenke C: Differential expression of MMP-2, MMP-9 and PCNA in endometriosis and endometrial carcinoma. Eur J Obstet Gynecol Reprod Biol: 74-78, 2012.

14. Hur SE, Lee JY, Moon HS and Chung HW: Angiopoietin-1, angiopoietin-2 and Tie-2 expression in eutopic endometrium in advanced endometriosis. Mol Hum Reprod 12: 421-426, 2006.

15. Wieser F, Cohen M, Gaeddert A, Yu J, Burks-Wicks C, Berga SL and Taylor RN: Evolution of medical treatment for endometriosis: Back to the roots? Hum Reprod Update 13: 487-499, 2007.

16. Yang M: Treatment of endometriosis by comprehensive TCM therapy: A clinical observation of 35 cases. New J Tradit Chin Med, 2004.

17. Tsai PJ, Lin YH, Chen JL, Yang SH, Chen YC and Chen HY: Identifying Chinese herbal medicine network for endometriosis: Implications from a population-based database in Taiwan. Evid Based Complement Alternat Med 2017: 7501015, 2017.

18. Gao H, Wang Z, Li Y and Qian Z: Overview of the quality standard research of traditional Chinese medicine. Frontiers of Medicine 5: 195-202, 2011.

19. Gong X, Lou J, Lu Q, Huang H and Jin Z: Bu Shen Huo Xue decoction restores endometrial leukemia-inhibitory factor but not Angiopoietin-2 expression, and improves uterine receptivity in the controlled ovarian stimulation rat model. Exp Ther Med 9: 751-757, 2015.

20. Zhang H, Li J, Sun W, Hu Y, Zhang G, Shen M and Shi X: Hyaluronic acid-modified magnetic iron oxide nanoparticles for MR imaging of surgically induced endometriosis model in rats. PLoS One 9: e94718, 2014.

21. Jiao L, Qi X, Lu G, Zhang Q, Zhang C and Gao J: Effect of traditional Chinese medicine (Xiaochaihu Tang) on the expression of MMP-2 and MMP-9 in rats with endometriosis. Exp Ther Med 6: $1385-1389,2013$ 
22. Reagan-Shaw S, Nihal M and Ahmad N: Dose translation from animal to human studies revisited. FASEB J 22: 659-661, 2008.

23. Yu J, Zhao L, Zhang D, Zhai D, Shen W, Bai L, Liu Y, Cai Z, Li J and $\mathrm{Yu} \mathrm{C}$ : The effects and possible mechanisms of puerarin to treat endometriosis model rats. Evid Based Complement Alternat Med 2015: 269138, 2015.

24. Livak KJ and Schmittgen TD: Analysis of relative gene expression data using real-time quantitative PCR and the 2(-Delta Delta $\mathrm{C}(\mathrm{T})$ ) method. Methods 25: 402-408, 2001.

25. Jiang HQ, Li YL and Zou J: Effect of recombinant human endostatin on endometriosis in mice. Chin Med J (Engl) 120: 1241-1246, 2007

26. Lucidi RS, Witz CA, Chrisco M, Binkley PA, Shain SA and Schenken RS: A novel in vitro model of the early endometriotic lesion demonstrates that attachment of endometrial cells to mesothelial cells is dependent on the source of endometrial cells. Fertil Steril 84: 16-21, 2005.

27. Anderson FW and Johnson CT: Complementary and alternative medicine in obstetrics. Int J Gynaecol Obstet 91: 116-124, 2005

28. Tindle HA, Davis RB, Phillips RS and Eisenberg DM: Trends in use of complementary and alternative medicine by US adults: 1997-2002. Altern Ther Health Med 11: 42-49, 2005.

29. Flower A, Liu JP, Lewith G, Little P and Li Q: Chinese herbal medicine for endometriosis. Cochrane Database Syst Rev: CD006568, 2012.

30. Giudice LC: Clinical practice. Endometriosis. N Engl J Med 362: 2389-2398, 2010.

31. McLaren J: Vascular endothelial growth factor and endometriotic angiogenesis. Hum Reprod Update 6: 45-55, 2000

32. Folkman J and Shing Y: Angiogenesis. J Biol Chem 267: 10931-10934, 1992.

33. Shifren JL, Tseng JF, Zaloudek CJ, Ryan IP, Meng YG, Ferrara N, Jaffe RB and Taylor RN: Ovarian steroid regulation of vascular endothelial growth factor in the human endometrium: Implications for angiogenesis during the menstrual cycle and in the pathogenesis of endometriosis. J Clin Endocrinol Metab 81: $3112-3118,1996$
34. Niedźwiecki S, Stepień T, Kopeć K, Kuzdak K, Komorowski J, Krupiński R and Stepień H: Angiopoietin 1 (Ang-1), angiopoietin 2 (Ang-2) and Tie-2 (a receptor tyrosine kinase) concentrations in peripheral blood of patients with thyroid cancers. Cytokine 36: 291-295, 2006.

35. Hur SE, Lee JY, Moon HS and Chung HW: Angiopoietin-1, angiopoietin-2 and Tie-2 expression in eutopic endometrium in advanced endometriosis. Mol Hum Reprod 12: 421-426, 2006.

36. He LL, Zhang WJ, Su H and Xu DG: Synergism between Ang-2 and VEGF and its application of anti-angiogenesis in tumor therapy - review. Zhongguo Shi Yan Xue Ye Xue Za Zhi 15: 445-448, 2007 (In Chinese).

37. Bohn KA, Adkins CE, Nounou MI and Lockman PR: Inhibition of VEGF and angiopoietin-2 to reduce brain metastases of breast cancer burden. Front Pharmacol 8: 193, 2017.

38. Yeniel AÖ, Erbas O, Ergenoglu AM, Aktug H, Taskiran D, Yildirim $\mathrm{N}$ and Ulukus M: Effect of oxytocin treatment on explant size, plasma and peritoneal levels of MCP-1, VEGF, TNF- $\alpha$ and histopathological parameters in a rat endometriosis model. Eur J Obstet Gynecol Reprod Biol 175: 134-139, 2014.

39. Liu S, Xin X, Hua T, Shi R, Chi S, Jin Z and Wang H: Efficacy of Anti-VEGF/VEGFR agents on animal models of endometriosis: A systematic review and meta-analysis. PLoS One 11: e0166658, 2016.

40. Hu C, Wang Z, Pang Z, Lu W, Cai X, Yang J, Wang D and Cao P: Guizhi fuling capsule, an ancient Chinese formula, attenuates endometriosis in rats via induction of apoptosis. Climacteric 17: 410-416, 2014. 